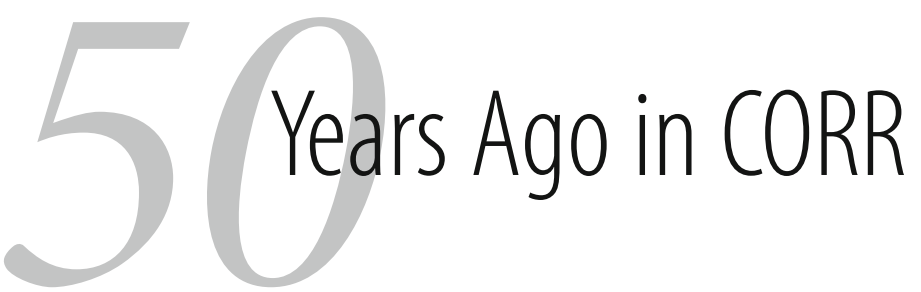

(C) The Association of Bone and Joint Surgeons 2008

\title{
The Iliac Apophysis: An Invaluable Sign in the Management of Scoliosis
}

\author{
Joseph C. Risser MD CORR 1958;11:111-119
}

isser reported in CORR in 1958 his well-known and important sign to assess the state of vertebral growth and predict which scoliosis curves would likely progress in growing children and which would not [4]. In an earlier article describing scoliosis progression in untreated patients [5], Risser and Ferguson had reported slow growth of the spine (sitting height increase of less than one-half inch) and slow increase in scoliosis deformity between the ages of 5 and 10 years (average increase in deformity of $4-5^{\circ}$ per year), and accelerated growth (one inch per year) and deformity (average $10^{\circ}$ increase per year) between the ages of $10-15$ years. In the article we highlight this month from 1958, Risser noted the growth plates in the spine were not easily visible and could not be used to reliably assess spinal growth [4]. However, "The completion of the excursion of the ossification of the iliac apophysis generally was coincident with that of the vertebral growth plates. Therefore, the attachment of the iliac apophysis has proved to be an excellent physiologic sign to indicate the completion of vertebral growth" [4].

Risser described the typical appearance of the iliac apophyseal ossification laterally and anteriorly on
Fig. 1A-B The illustrations show the US Risser grading system. (A) The US system comprises six grades. The US Risser 0 is defined as no ossification. The iliac wing is then divided into quarters, defining the first four grades $(1,2,3,4)$ of ossification of the apophysis (capping). The US Risser 5 begins when the ossified apophysis starts fusing to the wing at its posterior extremity. It continues during the whole process of apophysis fusion, which might takes as much as 2 years. (B) The French Risser grading system also comprises six stages. French Risser 0 is defined as no ossification. The iliac wing then is divided into thirds, defining the first three grades $(1,2,3)$ of ossification of the apophysis (capping). The fusion of the apophysis to the wing is divided into two grades: Risser 4, when the fusion commences posteriorly, and Risser 5, when the fusion is complete. (Reprinted with permission and CLippincott Williams and Wilkins, from Bitan FD, Veliskakis KP, Campbell BC. Differences in the Risser grading systems in the United States and France. Clin Orthop Relat Res. 2005;436:190-195.).

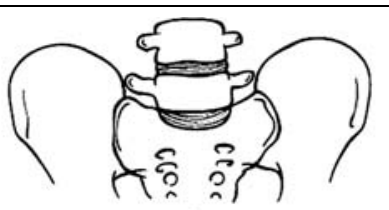

0

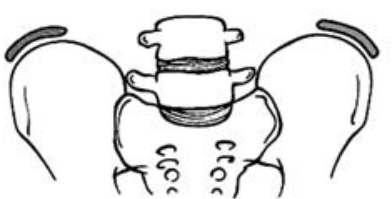

2

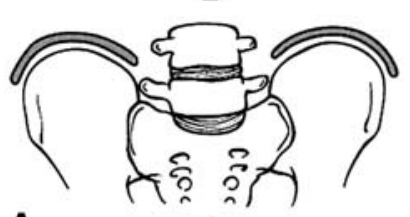

A

4

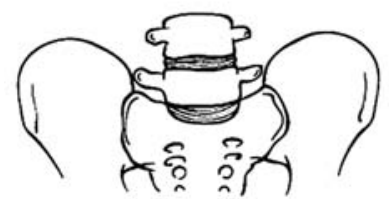

0

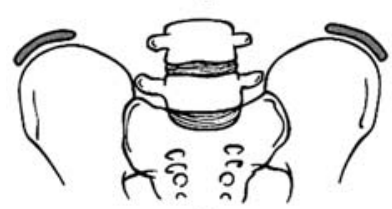

2

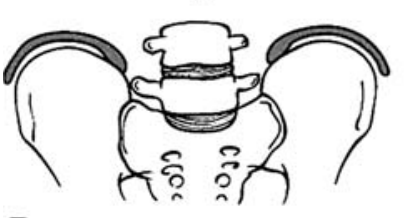

4

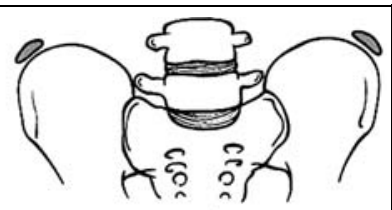

1

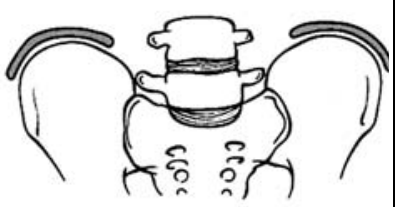

3

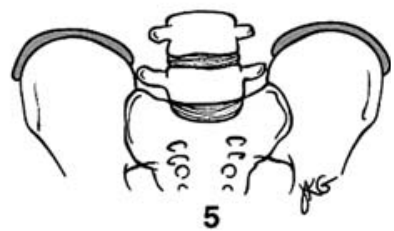

5
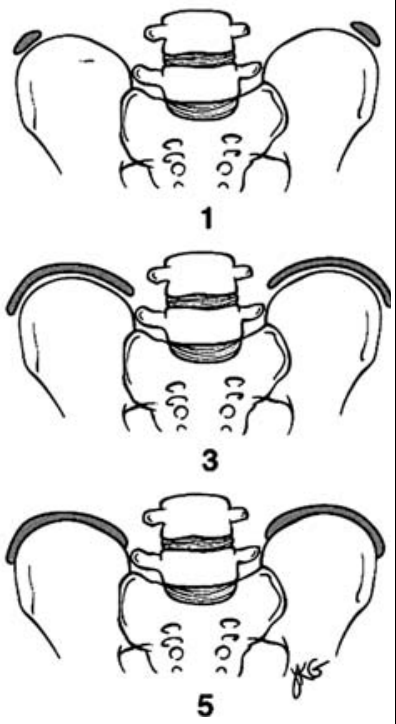
the crest, and used the term, "capping," to describe the progression. With continued growth the ossification extended posteriorly and eventually "dipped down to contact the ilium medially at its junction near the sacrum." With contact between the apophyseal ossification and the ilium, Risser considered the attachment "completed" and one could presume vertebral growth was also complete. This process typically took one year, but he noted completion as early as 7 months and as late as 3 years. In looking at the correlations of spinal growth, scoliotic deformity, and development of the excursion of the apophysis in 200 untreated patients, he further stated, "The coincidental development of the vertebral growth plates and the excursion of the ossification of the iliac apophysis is not 100 percent as seen in...3 cases." Deformities were more likely to increase rapidly prior to capping of the iliac apophysis, with a decrease in rate of deformity after completion [4].

According to Bitan et al. [1] Risser had initially described the sign 11 years earlier in a paper presented at the Annual Meeting of the American Academy of Orthopaedic Surgeons. Contemporary textbooks (e.g., The
Pediatric Spine [3]) refer to Risser Grades 1-4: Risser 1 occurs when the ossification first appears, Risser 2 when the ossification extends halfway across the iliac crest, Risser 3 when it extends three quarters across, and Risser 4 when completed (Fig. 1). Risser did not, however, describe those grades in his article. I have been unable to trace the origin of the grades, although according to Bitan et al. [1], "A grading system for ossification of the iliac apophysis (Risser sign) came into use in the mid 1950s in the United States and was adopted sometime later with modifications in France and other European countries." Thus, it appears use of the sign spread quickly and was perhaps even amplified with the four grades prior to its initial description in print. Clearly, Risser's basic observation came from one who is attentive to observations. Albert Szent-Gyorgyi, is supposed to have said, "Discovery consists of seeing what everybody has seen and thinking what nobody has thought" [2]. That would certainly apply to Risser's important observation.

Richard A. Brand MD Editor-in-Chief Clinical Orthopaedics and Related Research

\section{References}

1. Bitan FD, Veliskakis KP, Campbell BC. Differences in the Risser grading systems in the United States and France. Clin Orthop Relat Res. 2005;436:190-195.

2. Good I. The Scientist Speculates: An Anthology of Partly-Baked Ideas. London, UK: Heinemann; 1962.

3. McCarthy RE. Evaluation of the patient with deformity. In: Weinstein SL, ed. The Pediatric Spine. Philadelphia, PA: Lippincott Williams \& Wilkins; 2001:133-157.

4. Risser JC. The Iliac apophysis; an invaluable sign in the management of scoliosis. Clin Orthop Relat Res. 1958;11:111-119.

5. Risser JC, Ferguson AB. Scoliosis: its prognosis. J Bone Joint Surg Am. 1936;18:667-670.

50 Years Ago in CORR:

The Iliac Apophysis: An Invaluable Sign in the Management of Scoliosis Joseph C. Risser MD

Richard A. Brand MD 